\title{
Thermodynamic Phase Diagram of the Quantum Hall Skyrmion System
}

\author{
Kyungsun Moon \\ Department of Physics, Yonsei University, Seoul 120-749, Korea \\ Kieran Mullen \\ Department of Physics and Astronomy, University of Oklahoma, Norman, OK 73019
}

(September 4, 2018)

\begin{abstract}
We numerically study the interacting quantum Hall skyrmion system based on the Chern-Simons action. By noticing that the action is invariant under global spin rotations in the spin space with respect to the magnetic field direction, we obtain the low-energy effective action for a many skyrmion system. Performing extensive molecular dynamics simulations, we establish the thermodynamic phase diagram for a many skyrmion system.
\end{abstract}

PACS numbers: 75.10.-b, 73.20.Dx, 64.60.Cn 
The application of a strong magnetic field to a two dimensional electron gas "quenches" the kinetic energy of the electrons - removing it from consideration by constraining them to cyclotron motion. This allows other physical effects such as interactions and correlations to dominate. [1] When the number of electrons, $N_{e}$, is an integer multiple of the number of flux quanta, $N_{\phi}$, the groundstate of the system is a set of completely filled Landau levels. If we define the filling factor $\nu \equiv N_{e} / N_{\phi}$, then for $\nu=1,1 / 3$ and $1 / 5$ the groundstate is fully spin polarized. This polarization arises not simply from the Zeeman splitting but rather from interactions; indeed it persists even in the limit of zero Zeeman coupling. [2 [ The system minimizes its Coulomb energy by minimizing the overlap of electrons in the many-body wavefunction. When the electrons all have the same spin, the amplitude for two electrons to be at the same spot must vanish due to the exclusion principle, thereby reducing this electrostatic energy.

Near $\nu=1$ the low energy excitations of the system are distortions of the spin orientation, which are coupled to the local charge density. The system arranges spins of adjacent electrons so that they are nearly parallel, forming smooth distortions or textures in the spin field. This can be described by a non-linear sigma model, [3: 1 ] which predicts localized charged excitations [8 6] consisting of dimples in the spin field called skyrmions. [7] 9] It follows that if one moves away from $\nu=1$ the groundstate will include such textures as (possibly pinned) quasiparticles. This has been verified experimentally by NMR measurements of the spin polarization. [10,11] Other experiments such as optical absorption [12] and transport 13] are consistent with this picture.

At higher densities (i.e. further away from $\nu=1$ ) these quasiparticles themselves start to interact with each other. Each skyrmion has an overall U(1) symmetry corresponding to rotation about the z-axis. [14] When two are brought near each other, the lowest energy configuration is to align themselves anti-ferromagnetically. When many skyrmions interact, there is a competition between the Coulomb interaction, which favors a triangular lattice, and this antiferromagnetic $\mathrm{XY}$ interaction, which favors a bipartite one such as a square lattice. The resulting phase diagram is quite rich, and has been investigated theoretically. 
$15-17$

While the skyrmion lattice has not been seen directly, some experiments are suggestive. NMR measurements display a dramatic increase in the nuclear spin relaxation rate as a function of $|\nu-1|$, [1] and the low temperature heat capacity of multilayer 2DEG samples has a dramatic peak that may be related to the melting of a skyrmion lattice. 18 This anomalous, large $\left(\times 10^{5}\right)$ peak in the specific heat in GaAs quantum wells is postulated to come from a coupling of all of the nuclear magnetic moments of all the atoms adjacent to the quantum well, due to the symmetry breaking field of the Skyrme crystal.

In this paper we numerically study the phase diagram of the Skyrme crystal. Elsewhere we have derived a low-energy effective action, for the many body skyrmion system. [19] This effective action accurately describes the renormalization due to the higher Landau level mixing and is shown to be accurate for large and small skyrmion sizes. Here we use this action to variationally determine a two-body interaction potential between skyrmions. We then use this two-body interaction in a molecular dynamics (MD) simulation method to study the thermodynamic phase diagram of a many body interacting skyrmion system. Unlike earlier studies, we do not presume an a priori lattice structure. We incorporate the full, long range nature of the interaction allowing for competition between incompatible lattice structures and the possibility of frustration. At zero temperature, we find that the triangular lattice is formed below $\nu_{1} \cong 1.025$ and the square lattice becomes stable above $\nu_{1}$. At high filling factor, the square Skyrme crystal will melt due to strong quantum fluctuations. 114 Finite temperature simulation shows that at $\nu \cong 1.1$, the square Skyrme crystal melts at about $T_{m} \cong 0.035 \rho_{s}$. For the appropriate choice of experimental parameter, i.e. $\rho_{s} \cong 1.5 \mathrm{~K}$, $T_{m}$ is estimated to be $50 \mathrm{mK}$, which is very close to the value given by experiment. [18]

We begin with the effective action $\mathcal{S}_{E}$ for many skyrmion system 19

$$
\begin{aligned}
\mathcal{S}_{E}[\mathbf{m}] & =\frac{1}{2} \sum_{\mathbf{k}, \omega} V_{\text {eff }}(k)\left|J_{0}^{s}\right|^{2}+\sum_{\mathbf{k}, \omega} \frac{1}{2 \kappa}\left|\mathbf{J}^{s}\right|^{2} \\
& +\frac{\rho_{s}}{2} \int d \mathbf{r}(\nabla \mathbf{m})^{2}+\frac{1}{2} g \mu B m_{z}
\end{aligned}
$$




$$
+\frac{i}{\nu} \sum_{\mathbf{k}, \omega} \mathbf{A}^{(\mathbf{0})} \cdot \mathbf{J}^{s}-\frac{2 \pi}{\nu} \sum_{\mathbf{k}, \omega} \frac{1}{k^{2}} J_{0}^{s} \hat{z} \cdot \mathbf{k} \times \mathbf{J}^{s}
$$

where $\kappa=\bar{\rho} / m^{*}, V_{\text {eff }}(k)=V(k) /(1+b k)$ is a screened Coulomb interaction between skyrmions, $V(k)=2 \pi e^{2} / \epsilon k$ is a bare interaction, $\epsilon$ is the dielectric constant, $b=\ell^{2} / a_{B}$ with $a_{B}=\left(\hbar^{2} \epsilon / m^{*} e^{2}\right), \ell=(\hbar c /|e| B)^{1 / 2}$ is the magnetic length, and $\rho_{s}$ is the spin stiffness $e^{2} / 16 \sqrt{2 \pi} \epsilon$. The skyrmion three-vector $J_{\mu}^{s}$ is related to $\mathbf{m}(\mathbf{r})$, the local orientation of the spin texture via:

$$
J_{\mu}^{s}=\frac{\nu}{8 \pi} \epsilon_{\mu \nu \lambda}\left(\partial_{\nu} \mathbf{m} \times \partial_{\lambda} \mathbf{m}\right) \cdot \mathbf{m}
$$

By noticing that the presence of a finite Zeeman gap makes the skyrmion action invariant under global spin rotations by an angle $\phi$ in the XY-spin space [14], we chose to describe a skyrmion as a point particle with charge $q_{s}=\nu e$ and phase $\phi$. The Lagrangian for a many skyrmion system can then be simplified

$$
\begin{aligned}
\mathcal{L}\left[\mathbf{R}_{i}, \phi_{i}\right] & =\frac{1}{2} \sum_{i}\left\{M_{s}\left(\frac{d \mathbf{R}_{i}}{d t}\right)^{2}+I_{s}\left(\frac{d \phi_{i}}{d t}\right)^{2}\right\} \\
& -\frac{1}{2} \sum_{i \neq j} V\left(\mathbf{R}_{i}-\mathbf{R}_{j}, \phi_{i}-\phi_{j}\right) \\
& +\frac{i}{\nu} \sum_{\mathbf{k}} \mathbf{A}^{(\mathbf{0})} \cdot \mathbf{J}^{s}-\frac{2 \pi}{\nu} \sum_{\mathbf{k}} \frac{1}{k^{2}} J_{0}^{s} \hat{z} \cdot \mathbf{k} \times \mathbf{J}^{s} .
\end{aligned}
$$

The skyrmion transport mass $M_{s}$ and the moment of inertia $I_{s}$ are given by the following relations

$$
\begin{gathered}
M_{s}=\frac{2 \pi m^{*}}{\nu} \int_{\mathcal{S}} d \mathbf{r} \ell^{2}\left|J_{0}^{s}\right|^{2} \\
I_{s}=\frac{2 \pi m^{*} \ell^{2}}{\nu} \sum_{i=x, y} \int_{\mathcal{S}} d \mathbf{r}\left|\frac{\nu}{4 \pi}\left(\mathbf{m} \times \sigma_{y} \mathbf{m}_{\perp}\right) \cdot \partial_{i} \mathbf{m}\right|^{2}
\end{gathered}
$$

where $\mathcal{S}$ represents an area occupied by a single skyrmion and $\sigma_{y}$ is a Pauli spin matrix. The function $V(\mathbf{R}, \chi)$ is the interaction between two skyrmions, where $R=\left|\mathbf{R}_{i}-\mathbf{R}_{j}\right|$ is the relative distance and $\chi=\left(\phi_{i}-\phi_{j}\right)$ relative spin orientations. The last two terms in Eq.(3) take into account the exchange statistics of a skyrmion. [19] For small $\chi$, the two-body interaction can be decomposed into the following suggestive form 


$$
V[\mathbf{R}, \chi]=V_{0}(R)+V_{A}(R)[1+\cos (\chi)] .
$$

At large distances, $V_{0}(R)$ goes like $q_{s}^{2} / \epsilon R$ as a bare Coulomb repulsion between two point charges and $V_{A}(R)$ decays exponentially due to a finite Zeeman gap. Note that the angle dependent interaction $V_{A}(R)$ favors an antiferromagnetic spin alignment, i.e. $\chi=\pi$, trying to form a bipartite lattice, while $V_{0}(R)$ prefers a triangular lattice which does not belong to bipartite lattices. This two-body interaction is difficult to calculate variationally: A configuration of two skyrmions must be allowed to relax without allowing the skyrmions to migrate apart. We chose an ansatz that fixed the relative angle between two skyrmions to be $\chi$ and the distance to be $R$, and then calculated the minimum energy configurations numerically. Our variational form is:

$$
\omega\left(z, z^{*}\right)=\frac{z^{2}-\left(\frac{R}{2}\right)^{2}}{\lambda^{2}}\left[\frac{2 e^{i \theta(x) / 2}}{e^{-|z-R / 2| / 2 \xi}+e^{-|z+R / 2| / 2 \xi}}\right]
$$

where $z=x+i y$ denotes the position vector, $\xi=\left(2 \pi / E_{z}\right)^{1 / 2}$ with $E_{z}=(1 / 2) g \mu B / \rho_{s}$, and $\lambda$ is a variational parameter which controls the extent to which the spin fields are deformed. The spin fields $\mathbf{m}(\mathbf{r})$ are related to the complex field $\omega\left(z, z^{*}\right)$ via the following relation: $m_{x}+i m_{y}=2 \omega /\left(1+|\omega|^{2}\right)$. [7] When the distance $R$ is much larger than $\xi$, our variational ansatz describes two nearly free $\mathrm{n}=1$ skyrmions, and near $z=R / 2, \omega\left(z, z^{*}\right) \cong$ $\left[(z-R / 2) / \lambda_{s}\right] e^{|z-R / 2| / 2 \xi}$ with $\lambda_{s}=\lambda^{2} /(2 R)$ and $\theta(x)$ chosen to be 0 . The variational energy of an $n=1$ skyrmion based on the above ansatz yields 1.789 in the units of $4 \pi \rho_{s}$ and $\lambda_{s}=2.34 \ell$, which is remarkably close to the exact value 1.788 from the field theoretic action. 19 In the opposite limit of $R=0$, it depicts an $n=2$ skyrmion, whose variational energy is calculated to be 3.939 , which is very close to the exact value of 3.913 in the units of $4 \pi \rho_{s}$, which lends a strong credence to our variational ansatz. By minimizing the action in Eq.(11) for the proposed two skyrmion ansatz with $\theta(x)=0$, the two-body interaction $V_{0}(R)$ is calculated. In order to calculate the angle-dependent interaction $V_{A}(R)$, we adjust $\lambda$ to minimize the energy for the following ansatz for the gradual spin twist $\theta(x)$, which rotates m with respect to the $\hat{z}$ axis:

$$
\theta(x)=\theta_{0} \tanh (2 x / R)
$$


which uniformly inserts twist between the two skyrmions. Here $\pi-\theta_{0}$ is the relative phase difference $\chi$ between two skyrmions. This ansatz does not change the Zeeman energy, since $m_{z}$ stays the same. In Fig.(11) we plot the interaction strengths $V\left(R, \pi-\theta_{0}\right)$ as a function of distance $R$ and relative angle $\theta_{0}$ between the textures. We can break this into two parts as described in Eq.(6) and the interaction profile thus obtained is given by

$$
\begin{gathered}
V_{0}(R) \cong 3.578+\frac{0.35+0.175 R}{1+0.0213 R+0.0147 R^{2}} \\
V_{A}(R) \cong 0.629 e^{-0.434 R}
\end{gathered}
$$

where $V_{0}$ and $V_{A}$ are in the units of $4 \pi \rho_{s}, R$ in the unit of $\ell$. One can notice that $V_{0}(R)$ bends over to a certain finite value as $R$ decreases due to the soft core nature of skyrmion and $\partial^{2} V_{0} / \partial R^{2}$ becomes negative at $\nu_{c} \cong 1.2$. This leads to a complex pattern at filling factors around and above $\nu_{c}$ [20], which will most likely be preempted by quantum melting. 114 Based on the interactions obtained above, we employ overdamped molecular dynamics simulations at finite temperature

$$
\begin{aligned}
\eta_{R} \frac{d \mathbf{R}_{i}}{d t}= & -\sum_{j \neq i} \partial_{\mathbf{R}_{i}} V_{0}\left(\mathbf{R}_{i j}\right) \\
& -\sum_{j \neq i} \partial_{\mathbf{R}_{i}} V_{A}\left(\mathbf{R}_{i j}\right)\left[1-\cos \left(\phi_{i j}-\pi\right)\right]+\vec{\xi}_{i}^{R}(t) \\
\eta_{\phi} \frac{d \phi_{i}}{d t}= & -\sum_{j \neq i} V_{A}\left(\mathbf{R}_{i j}\right) \sin \left(\phi_{i j}-\pi\right)+\xi_{i}^{\phi}(t) \\
& \left\langle\xi_{i}^{\alpha}(t) \xi_{j}^{\alpha}\left(t^{\prime}\right)\right\rangle=2 \eta_{\alpha} T \delta_{i j} \delta\left(t-t^{\prime}\right) .
\end{aligned}
$$

Here $\eta_{\alpha}$ is the damping parameter with $\alpha=R, \phi, \mathbf{R}_{i j}=\mathbf{R}_{i}-\mathbf{R}_{j}, \phi_{i j}=\phi_{i}-\phi_{j}$, and $\xi_{i}^{\alpha}(t)$ is a Gaussian white noise which represents the thermal fluctuations following the fluctuationdissipation theorem. It can also help the system to reach the true ground state by providing a random noise.

The number of skyrmions $N$ was fixed to be 576 and the mean particle spacing $R_{s}$ varies with $\nu$ as $(2 \pi /(\nu-1))^{1 / 2}$ assuming the square lattice structure. Now we construct 
the phase diagram in the filling factor-temperature plane. The initial configurations are chosen randomly. We let the MD simulations evolve with time, make sure that the system reaches the equilibrium state, then freeze the skyrmion configuration and measure the static structure factor $S(\mathbf{k})$

$$
S(\mathbf{k})=\frac{1}{N^{2}} \sum_{i, j} e^{i \mathbf{k} \cdot\left(\mathbf{R}_{i}(t)-\mathbf{R}_{j}(t)\right)}
$$

By measuring $S(\mathbf{k})$ and visually checking the snap shots, we identify the corresponding phases. First we have studied the zero temperature phase diagram. Insets of Fig.(2) demonstrate $S(\mathbf{k})$ at $\nu=1.02$ and 1.07 exhibiting the six and four-fold peaks, as expected for the triangular and square lattice structures, respectively. At zero temperature, with the increase of filling factor away from $\nu=1$, the system initially forms a triangular lattice and above a critical value of $\nu_{1} \cong 1.025$ a square lattice. At higher densities, the square Skyrme crystal will melt due to strong quantum fluctuations. [14,20] Subsequently, we investigate the melting lines for the various Skyrme crystals via finite temperature MD simulations. The melting phase boundary can be established by measuring the peak heights of static structure factor $S\left(\mathbf{K}_{0}\right)$ as a function of temperature and $\mathbf{K}_{0}=(2 \pi, 2 \pi)$ for the square Skyrme crystal. The Lindemann criterion says that the solid melts when the thermal fluctuations of the equilibrium positions are about $15 \%$ of the mean particle spacing $R_{s}$, that is, $\sqrt{<\mathbf{u}^{2}>} \cong c_{L} R_{s}$ with $c_{L} \cong 0.15$. Assuming Gaussian fluctuations, we obtain that $S\left(\mathbf{K}_{0}\right) \cong e^{-\mathbf{K}_{0}^{2}<\mathbf{u}^{2}>/ 2} \cong 1 / 3$. [21] Thus when the peak height of $S\left(\mathbf{K}_{0}\right)$ reaches about $1 / 3$, we consider the crystal having melted. In Fig.(2), we have plotted the melting phase boundary and noticed that at around $\nu=1.1$, the melting temperature is about $0.035 \rho_{s}$. The experimental value of $\rho_{s}$ is about $1.5 \mathrm{~K}$ yielding $T_{m} \cong 50 \mathrm{mK}$, which is very close to the experimentally suggestive melting temperature. The spin structure factor $S_{\phi}(\mathbf{k})$ has also been measured

$$
S_{\phi}(\mathbf{k})=\frac{1}{N^{2}} \sum_{i, j} e^{i \mathbf{k} \cdot\left(\mathbf{R}_{i}(t)-\mathbf{R}_{j}(t)\right)} e^{i\left(\phi_{i}(t)-\phi_{j}(t)\right)} .
$$

Within the square Skyrme crystal phase, our simulations clearly demonstrate that the system possesses an antiferromagnetic spin order by exhibiting the peaks of $S_{\phi}(\mathbf{k})$ at $\mathbf{k}=(\pi, \pi)$. 20 
We note that although it is possible that there could be a regime where the triangular and square lattices could compete to produce a disordered phase, we do not observe this with our model using the experimentally relevant parameters. In order to clarify this interesting possibility, further studies including careful finite size scaling analysis is required. [20] In this work, we do not treat the terms in the action that guarantee the fermionic nature of skyrmions, nor do we include any quantum dynamics in the MD simulation. 222 Finally, we ignore any three-body interactions, treating the spin texture action as a set of pairwise interactions between skyrmions.

In summary, we have determined an approximate two body interaction potential for skyrmions from an effective action via a variational calculation. Using this potential we

have performed molecular dynamics simulations that have determined the phase diagram of the skryme system without having to posit a crystal structure. We find that the melting temperature within our model is close to the specific heat peaks seen in proposed skyrme crystal experiments, which gives a strong support to the very existence of skyrme crystal.

\section{ACKNOWLEDGMENTS}

It is our great pleasure to acknowledge S.M. Girvin, H. Fertig, and D.H. Lee for useful discussions. K. Moon wishes to acknowledge the financial support of the Korea Research Foundation made in the program year of 1998. This work was supported in part by Yonsei University Research Fund of 1998 and NSF grant DMR-9502555. 


\section{REFERENCES}

[1] R. E. Prange and S. M. Girvin, The Quantum Hall Effect, (Springer-Verlag, New York, 1988).

[2] D. H. Lee and C. L. Kane, Phys. Rev. Lett. 64, 1313 (1990).

[3] S. L. Sondhi, A. Karlhede, S. A. Kivelson and E. H. Rezayi, Phys. Rev. B 47, 16419 (1993).

[4] K. Moon, H. Mori, K. Yang, L. Belkhir, S. M. Girvin, A. H. MacDonald, L. Zheng, D. Yoshioka, and S. C. Zhang, Phys. Rev. B 51, 5138 (1995).

[5] A. H. MacDonald, H. A. Fertig, and L. Brey, Phys. Rev. Lett. 76, 2153 (1996).

[6] M. Abolfath, J. J. Palacios, H. A. Fertig, S. M. Girvin and A. H. MacDonald, Phys. Rev. Lett. 56, 6795 (1997).

[7] R. Rajaraman, Solitons and Instantons, (North-Holland, Amsterdam, 1982).

[8] M. Rasolt, B. I. Halperin, and D. Vanderbilt, Phys. Rev. Lett. 57, 126 (1986).

[9] S. M. Girvin and A. H. MacDonald, in Novel Quantum Liquids in Low-Dimensional Semiconductor Structures, edited by S. Das Sarma and A. Pinczuk (Wiley, New York, 1996).

[10] S. E. Barrett, G. Dabbagh, L. N. Pfeiffer, K. W. West, and R. Tycko, Phys. Rev. Lett. 74, $5112(1995)$.

[11] R. Tycko, S. E. Barrett, G. Dabbagh, L. N. Pfeiffer, and K. W. West, Science 268, 1460 (1995).

[12] E. H. Aifer, B. B. Goldberg, and D. A. Broido, Phys. Rev. Lett. 76, 680 (1996).

[13] A. Schmeller, J. P. Einsenstein, L. N. Pfeiffer, and K. W. West, Phys. Rev. Lett. 75, $4290(1995)$.

[14] R. Coté, A. H. MacDonald, L. Brey, H. A. Fertig, S. M. Girvin and H. T. C. Stoof, Phys. Rev. Lett. 78, 4825 (1997).

[15] L. Brey, H. A. Fertig, R. Coté, and A. H. MacDonald, Phys. Rev. Lett. 75, 2562 (1995).

[16] A. G. Green, I. I. Kogan, and A. M. Tsvelik, Phys. Rev. B 54, 16838 (1996). 
[17] M. Rao, S. Sengupta, and R. Shanar, Phys. Rev. Lett. 79, 3998 (1997).

[18] V. Bayot, E. Grivei, J.-M. Beuken, S. Melinte, M. B. Santos and M. Shayegan, Phys. Rev. Lett. 76, 4584 (1996): ibid 79, 1718 (1997).

[19] K. Moon and K. Mullen, Phys. Rev. B 57, 14833 (1998).

[20] K. Moon et al., unpublished.

[21] S. Scheidl and V. Vinokur, Phys. Rev. B 57, 13800 (1998).

[22] For sufficiently small filling factors, exchange contributions are shown to be negligible for two-dimensional Wigner crystals : H. Yi and H. A. Fertig, Phys. Rev. B 58, 4019 (1998). 


\section{FIGURES}

FIG. 1. The two-body interaction potential as a function of $R$ and $\theta_{0}$, as determined by variational calculations of the total energy. The curve with $\theta_{0}=0$ represents $V_{0}(R)$. Note the steep dependence of the energy as a function of twist.

FIG. 2. Thermodynamic phase diagram of a manybody skyrmion system: Below $\nu_{1} \sim 1.025$, triangular lattice is stable. Above $\nu_{1}$, square lattice is formed, which melts at around $0.035 \rho_{s}$ for $\nu=1.1$. At some higher density, square skyrme crystal will melt due to quantum fluctuations. Insets show static structure factor $S(\mathbf{k})$ : At filling factor $\nu=1.02$, six-fold peaks appear at the corresponding points to the triangular lattice. At filling factor $\nu=1.07$, four-fold peaks appear at the corresponding points to the square lattice. 


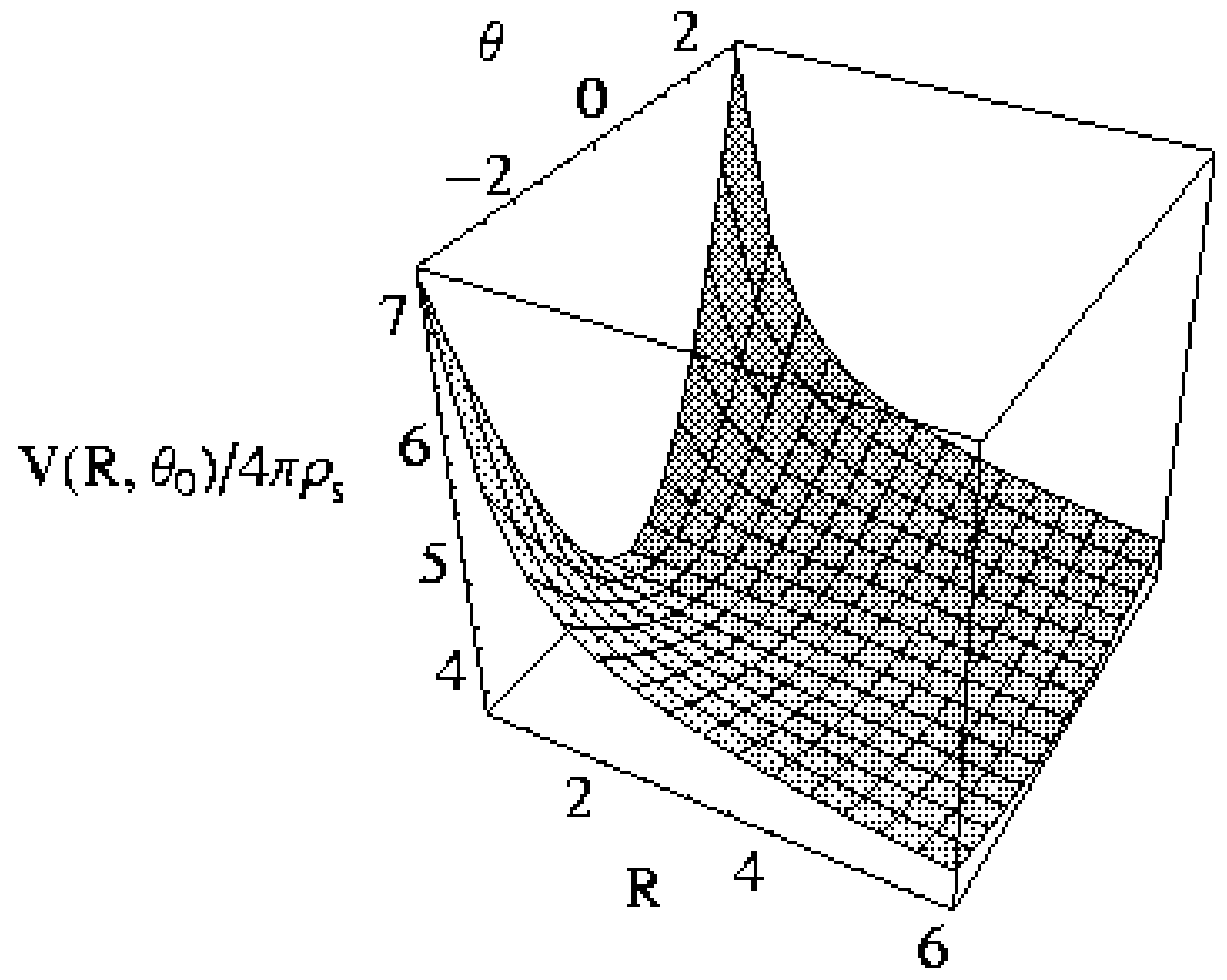




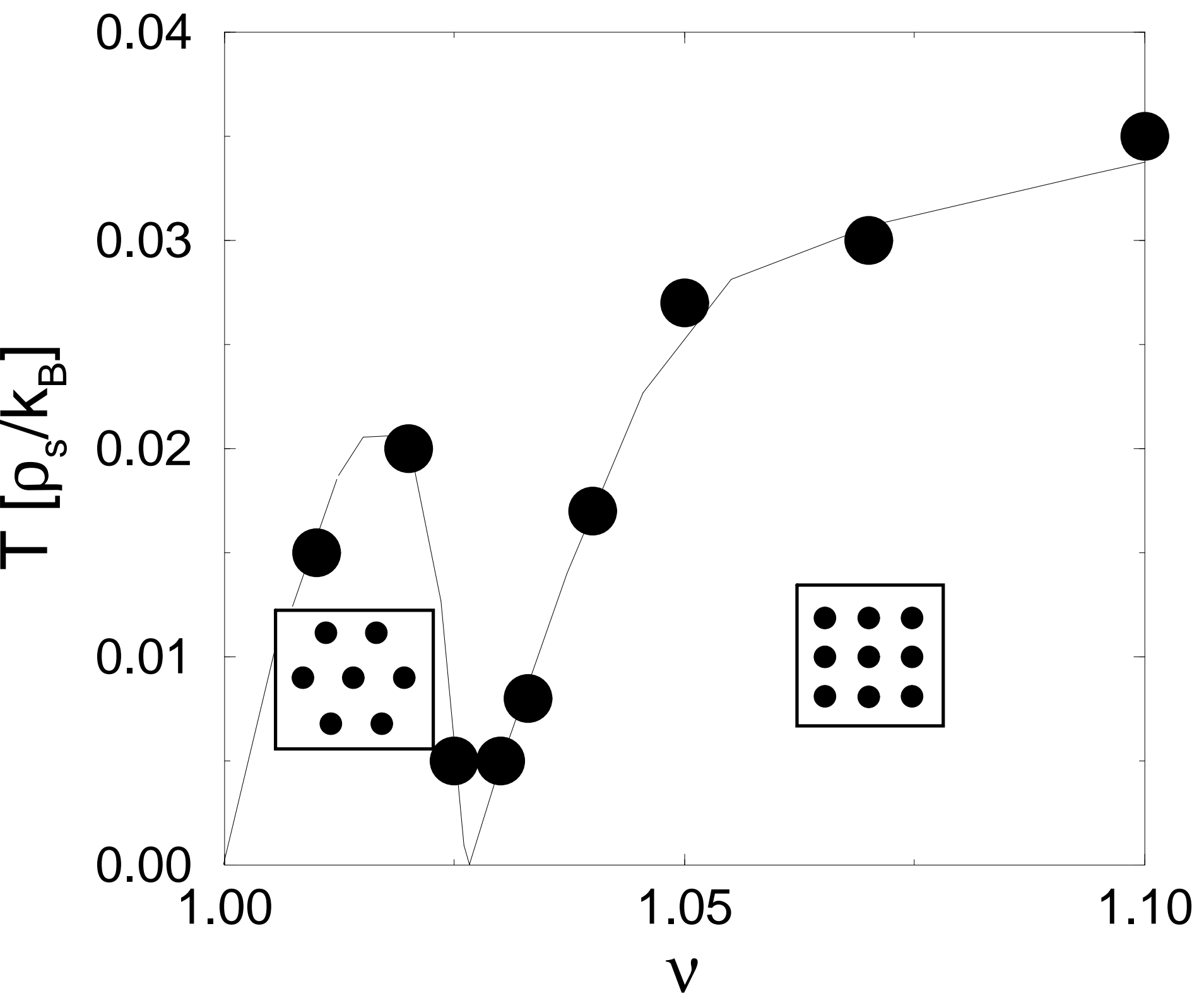

